\title{
Competencias laborales de trabajadores agrícolas de la Región del Libertador Bernardo O’Higgins, Chile. Un estudio de caso
}

\author{
Labor competitions of agricultural workers of the region \\ Libertador Bernardo O'Higgins, Chile. A case study
}

\author{
David Mella Osorio ${ }^{1}$, Ma. Beatriz Vera Oyarzún ${ }^{1 *}$, Patricia Bahamonde Brintrup ${ }^{1}$
}

\begin{abstract}
RESUMEN
En Chile, durante agosto del año 2008 entró en vigencia la Ley 20.267 que crea el Sistema Nacional de Certificación de Competencias Laborales y perfecciona el estatuto de capacitación y empleo. Los procesos de evaluación y certificación permiten conocer el nivel de competencia de las personas respecto de un mínimo satisfactorio predefinido por un estándar. Dichas normas se denominan Unidades de Competencia Laborales (UCL), las que están asociadas a perfiles ocupacionales, representativos de diferentes sectores productivos del país. El objetivo de la presente investigación es analizar las condiciones de cumplimiento de las competencias básicas, conductuales y funcionales existentes entre el nivel de competencia de los trabajadores del sector agrícola, subsector frutícola, según los perfiles establecidos por la Fundación para el Desarrollo Frutícola (FDF), en cuatro empresas de la Región del Libertador Bernardo O’Higgins. La investigación determinó los niveles de competencias de 82 trabajadores distribuidos en cuatro empresas del rubro agroindustrial de la región Libertador Bernardo O'Higgins y analizó las distintas unidades de competencias laborales tanto básicas, genéricas y conductuales, como técnicas que ejerce cada trabajador en su puesto de trabajo. Los resultados mostraron que el análisis entre empresas no muestra diferencias significativas en el nivel de competencias de los trabajadores, ni en los distintos perfiles ocupacionales evaluados. El $90 \%$ de los trabajadores están por sobre el estándar o mínimo exigido en el programa de certificación de competencias laborales, para ser un trabajador competente en su labor. En las cuatro empresas existen personas que se ubican sobre el promedio, destacando en su labor respecto de las exigencias técnicas exigidas, pero nadie destaca en la unidad de competencia asociada a aspectos transversales del ámbito laboral, como higiene y seguridad, reglamento interno y conducta del trabajador, etc. El perfil de embalador de fruta de exportación representa un $63,4 \%$ del total de la muestra, lo que releva la gran importancia de este perfil para las empresas del sector, siendo las principales actividades la selección, manipulación y envasado de la fruta procesada.
\end{abstract}

Palabras clave: competencia laboral, trabajador agrícola, buenas prácticas agrícolas.

\begin{abstract}
The law 20.267 became valid in August 2008 which creates the National System of Certifications for work skills and improvement of the job trainee. The process of evaluation and certification allows us to know a person's skills level regarding to a predefined standard. This rule is known as the "Unidades de Competencia Laborales (UCL)" which in english means Labor Skills Unit, which are associated to occupational profiles, representative of different productive segments of the country. This investigation determined the skills levels of 82 employees distributed in 4 companies of the Agricultural Industry Business of the region of Libertador Bernardo O'Higgins and it analyzed the different labor skills units based in basics, generics and behavioral marks, as well as techniques which every employee show in their job position. The results showed that the analysis between companies does not show significant differences in the work skills level of the employee between companies or in the different occupational profiles evaluated. In all the companies chosen for this investigation, the $90 \%$ of the employees are above the standard or minimum demanded in the program of certification of labor work skills in order to be a competent worker in their job position. There are people above the media in the 4 companies of the study, with emphasis in their techniques, but nobody in the working skills units related to the basic part,such as hygene and security, internal rules and behavior of the employee. The profile of export fruit packer represents $63.4 \%$ of the total sample, which reveals the importance of this profile for the companies in the sector, being the main activities the selection, handling and packaging of processed fruit.
\end{abstract}

Key words: labor competition, agricultural worker, good agricultural practices.

1 Instituto de Economía Agraria, Facultad de Ciencias Agrarias de la Universidad Austral de Chile. Independencia 631, Valdivia, Chile.

* Autor para correspondencia: bvera@uach.cl

Fecha de Recepción: 2 Diciembre, 2014.

Fecha de Aceptación: 3 Marzo, 2015. 


\section{Introducción}

La apertura a los mercados internacionales, mediante los tratados de libre comercio con sectores que representan importantes naciones de todo el mundo, principalmente Norteamérica, la Comunidad Europea y el Asia, hace que la economía chilena sea cada vez más abierta y dinámica; lo que ha provocado el continuo desarrollo de las empresas y en especial la agroindustria. Como resultado de ello, prevalece la gran inserción de productos chilenos en el extranjero y demandas cada vez más crecientes de mano de obra. Su ingreso no ha sido simple, al contrario, la tarea de satisfacer los elevados estándares de calidad establecidos por estos mercados involucran la compleja tarea de cumplir con múltiples requerimientos, que van desde los aspectos laborales, ambientales, administrativos, hasta la certificación a nivel predial y agroindustrial.

En la actualidad, es cada vez más importante internalizar y analizar la trascendencia que tiene para la agroindustria, el desarrollo del recurso humano, a pesar de que las principales normas de certificación, como Buenas Prácticas Agrícolas (BPA), Global Gap y Tesco Nature's Choice (TNC) a nivel predial y Buenas Prácticas de Manufactura (BPM), Hazard analysis and critical control points (HACCP), British Retail Consortium (BRC), e International Organization for Standardization (ISO), no la exigen aún, es de vital importancia anticiparse a tener mano de obra capacitada en las labores que involucran el procesamiento.

Según señalan De Ferranti et al. (2002), "aumentar la productividad es esencial para mejorar las posibilidades del crecimiento, la habilidad para dominar las destrezas y la tecnología es fundamental para incrementar la productividad". A su turno, las destrezas adquiridas son productivas solamente "si van de la mano con equipos de alta tecnología, con la adaptación de tecnologías avanzadas y con otras inversiones que tienen lugar en una economía con incentivos para crecer".

Por su parte, el Estado de Chile ha generado políticas públicas para el desarrollo de capital humano en el sector agrícola, con el fin de aumentar la competitividad y empleabilidad del sector productivo. Este esfuerzo ha sido mancomunado entre el sector público y privado; y está siendo impulsado por distintas instituciones, como el Servicio Nacional de Capacitación y Empleo (SENCE), ChileValora, Fundación Chile, Asociación de Exportadores de Fruta A.G. (ASOEX) y la Fundación para el Desarrollo Frutícola (FDF).

Dichas políticas se han enfocado en torno a la generación de Perfiles Ocupacionales acordes a las necesidades actuales de la industria, las que además, han sido homologadas en un principio por el programa Chilecalifica, retomado por Fundación Chile y en estos momentos actualizados y validados por el programa ChileValora. Todo esto, con el fin de diseñar un sistema de certificación de calidad que asegure que sean estandarizados y homologados a nivel nacional y ser un aporte para la empresa y el trabajador.

La ley 20.267, que crea el Sistema Nacional de Certificación de Competencias Laborales, el que perfecciona el estatuto de capacitación y empleo, es promulgada el 6 de junio 2008 y publicada el 25 de junio del mismo año. El organismo ejecutor es el Ministerio del Trabajo y Previsión Social; Subsecretaría del Trabajo (Ministerio del Trabajo y Previsión Social, 2008).

A diferencia de otros países, en Chile la preocupación por las competencias laborales surge desde el sector productivo. La exigencia de una mayor calidad en la calificación laboral se convierte en la dimensión de mayor relevancia en los procesos de competitividad nacional e internacional, siendo uno de los detonantes importantes los Tratados de Libre Comercio suscritos por el país, que obligan a tomar medidas para no quedar fuera del mercado (Servicio Nacional de Capacitación y Empleo -SENCE-, 2008).

Según Etiennette (2002), el enfoque de las competencias crece en importancia en diversas partes del mundo, ocupando un papel cada vez más central en la capacitación y abarcando niveles de la educación formal, hasta la educación superior.

Arguelles (1999) indica que la formación y capacitación de recursos humanos no deben ser procesos finitos y de corta duración, sino largos y continuos durante toda la vida productiva de la persona.

Chilecalifica transversaliza el enfoque de las competencias laborales a la educación formal, no formal y experiencia laboral, construidas por medio de una serie de proyectos puestos en marcha, donde tiene directa participación Fundación Chile y el Servicio Nacional de Capacitación y Empleo. Los cuales constituyen los antecedentes de lo que será el Sistema de Formación Permanente (Chile, Servicio Nacional de Capacitación y Empleo-SENCE-, 2008). 
La Organización para la Cooperación y el Desarrollo Económico (OCDE) ha propuesto definir las competencias como "la capacidad para responder exitosamente a una demanda compleja o llevar a cabo una actividad o tarea, incluyendo las actitudes, valores, conocimientos y destrezas que hacen posibles la acción efectiva" (Fundación Chile, 2004).

El sistema nacional de competencias laborales tiene por objeto el reconocimiento formal de las competencias del trabajador, independientemente de la forma en que hayan sido adquiridas y si tienen o no un título o grado académico, otorgado por la enseñanza formal de conformidad a las disposiciones de la ley $\mathrm{N}^{\circ} 18.962$, Orgánica Constitucional de Enseñanza; así como favorecer las oportunidades de aprendizaje continuo de las personas, su reconocimiento y valorización (Ministerio del Trabajo y Previsión Social, 2008).

El perfil ocupacional es el resultado del levantamiento de competencias laborales en un sector productivo, donde se definieron funciones críticas y se estandarizaron las capacidades asociadas a dichas funciones. Todo el proceso de definición tiene como componente esencial la observación y discusión en terreno, por parte de un grupo técnico, sobre las buenas prácticas y los mejores desempeños de aquellas funciones laborales y de los criterios con que se medirán estos. La persona demuestra en la práctica si es "competente" o "aún no competente" (Servicio Nacional de Capacitación y Empleo-SENCE-, 2008).

Dentro del sistema de educación, el perfil ocupacional juega un rol específico permanente, porque sirve de referente para acometer la formulación de currículo, estandarizar las competencias establecidas en el perfil, los aprendizajes a ser medidos, la metodología para la implementación de dichos aprendizajes, el material de apoyo pertinente, las condiciones en que se realizarán las actividades y los perfiles de los relatores, otorgando pertinencia porque se basa en las necesidades del sector productivo y calidad al ser construido con el enfoque de competencias laborales (Servicio Nacional de Capacitación y Empleo -SENCE-, 2008).

Dentro de los perfiles ocupacionales se encuentran las Unidades de Competencia Laboral (UCL), las que son un estándar que describe los conocimientos, las habilidades y aptitudes que un individuo debe ser capaz de desempeñar y aplicar en distintas situaciones de trabajo, incluyendo las variables, condiciones o criterios para inferir que el desempeño fue efectivamente logrado (Ministerio del Trabajo y Previsión Social, 2008).

La evaluación de competencias laborales es un proceso de verificación del desempeño laboral de una persona con un estándar previamente homologado $\mathrm{y}$ validado por un programa gubernamental. $\mathrm{La}$ certificación de competencias es un proceso de reconocimiento formal, por una entidad independiente, de las competencias laborales demostradas por un individuo en el proceso de evaluación (Ministerio del Trabajo y Previsión Social, 2008).

Las competencias laborales en el sector agroalimentario tienen la importancia de aportar valor agregado a sus exportaciones y productos nacionales, además, estas empresas tienen gran implicancia social en la generación de empleos (Fundación Chile, 2007).

Por otra parte, el sector agrícola exhibe el nivel de calificación más bajo a nivel nacional, a pesar de ser uno de los más expuestos a nuevas exigencias del exterior en materia de capital humano. Esto convierte a las iniciativas de certificación y desarrollo de competencias laborales, en aspectos claves para el posicionamiento global del sector (Fundación Chile, 2007).

El objetivo de la presente investigación es analizar las condiciones de cumplimiento de las competencias básicas, conductuales y funcionales existentes entre el nivel de competencia de los trabajadores del sector agrícola, subsector frutícola, según los perfiles establecidos por la Fundación para el Desarrollo Frutícola (FDF), en cuatro empresas de la Región del Libertador Bernardo O'Higgins.

\section{Materiales y Métodos}

En el presente estudio se analizó el nivel de competencia de trabajadores del sector agrícola, según los perfiles establecidos por Fundación para el Desarrollo Frutícola (FDF) 2010, en cuatro empresas de la Región del Libertador Bernardo O’Higgins, Chile.

\section{Universo y Muestra del Estudio}

Consideró 82 trabajadores distribuidos entre sector agrícola, subsector frutícola (packings) de la Región del Libertador Bernardo O’Higgins. 
El estudio práctico se realizó entre noviembre de 2010 y abril de 2011. En este período se elaboraron y aplicaron los instrumentos de evaluación para reunir la información requerida en la investigación.

\section{Metodología}

Cada instrumento de evaluación aportó al final del proceso una ponderación al promedio del candidato, si este presenta un nivel mayor o igual a $75 \%$, es señalado como un trabajador "competente" en las UCL evaluadas. Los resultados del análisis estadístico de cada UCL fueron obtenidos por medio de la aplicación del software estadístico SPSS (Statistical Package for the Social Sciences), en su versión 11.5.

Según Fundación Chile (2010), el proceso de evaluación de competencias laborales utiliza los siguientes instrumentos obligatorios y optativos.

\section{Instrumentos Obligatorios}

Ficha datos personales. Es un documento donde se reúnen antecedentes personales del candidato y tienen que tener mínimo una temporada de experiencia en su labor.

Carta compromiso. Es un documento que tiene como finalidad que el trabajador establezca un compromiso con el proyecto.

Set de observación en terreno (SOT). Este contiene información de las diferentes Unidades de Competencias Laborales (UCL), que realiza el trabajador en su labor diaria. De acuerdo con la actividad que realizan los candidatos, se evaluaron un mínimo de dos UCL, la primera es transversal para todos los perfiles y se denomina "Respetar Normas de Higiene y Seguridad" (Código SAO 001 o conductuales), la que es de carácter obligatoria en todas las evaluaciones en terreno, y la segunda UCL evaluada es técnica y dependerá de la labor específica que el trabajador realice en el momento de la evaluación en terreno. Se realizaron tres observaciones en terreno diferidas en aproximadamente una semana cada una, con el objetivo de ver al trabajador en diferentes circunstancias que pudieran afectar su quehacer diario y tener una visión objetiva de su desempeño en terreno. En la Tabla 1 se indica la escala utilizada.

Autoevaluación (AUE). Es la evaluación de parte del candidato sobre la labor realizada y el
Tabla 1. Desarrollo de la competencia en terreno.

\begin{tabular}{ccc}
\hline Nota & $\%$ & Nivel de desarrollo de la competencia \\
\hline 1 & 25 & MÍNIMO \\
2 & 50 & EN DESARROLLO \\
3 & 75 & DESARROLLADO \\
4 & 100 & EXCEPCIONAL \\
\hline
\end{tabular}

Fuente: Chile, Fundación Chile (2010).

cumplimiento de las normas de higiene y seguridad del perfil ocupacional, tiene una ponderación en el promedio de la evaluación final del candidato, este instrumento es obligatorio en la evaluación.

Entrevista de conocimientos. Es un set de preguntas elaborado por la Fundación para el Desarrollo Frutícola (FDF), que se aplica al trabajador en terreno, estas preguntas tienen relación con las UCL evaluadas, por lo que se realizan igual cantidad de entrevistas que UCL consideradas.

\section{Instrumentos Optativos}

Simulación de habilidades y conocimientos. Se aplica en el caso que en terreno no se pueda observar una situación descrita en el SOT.

Evaluación del jefe directo (EJD). Evaluación de parte del jefe o supervisor directo en terreno o el que tiene más contacto con el candidato sometido a observación.

Retroalimentación. Instrumento que no tiene ponderación en el promedio final.

Para procesar la información obtenida existen distintas ponderaciones en el promedio final de la evaluación, dependiendo del perfil ocupacional evaluado, siendo el de mayor ponderación el SOT, que aplica un evaluador externo previamente capacitado por Fundación Chile, el cual equivale entre $60 \%$ y $65 \%$ del promedio final.

\section{Resultados y Discusión}

\section{Competencias Básicas, Conductuales y Funcionales}

Según SENCE 2008 existen varias tipologías de competencias, pero en la literatura describen principalmente tres tipos que se detallan en el Tabla 2.

Según el Ministerio del Trabajo y Previsión Social (2008), una competencia laboral son las aptitudes, conocimientos y destrezas necesarias para cumplir exitosamente las actividades que componen 
Tabla 2. Tipología de competencias.

\begin{tabular}{lll}
\hline \multicolumn{1}{c}{ Básicas } & \multicolumn{1}{c}{$\begin{array}{c}\text { Conductuales } \\
\text { (genéricas) }\end{array}$} & \multicolumn{1}{c}{$\begin{array}{c}\text { Funcionales } \\
\text { (técnicas) }\end{array}$} \\
\hline $\begin{array}{l}\text { Mínimas para desempeñarse en } \\
\text { cualquier ámbito de desarrollo } \\
\text { personal y laboral. }\end{array}$ & $\begin{array}{l}\text { Corresponden a comportamientos y } \\
\text { actitudes transversales a diferentes } \\
\text { ámbitos de actividad personal y laboral. }\end{array}$ & $\begin{array}{l}\text { Capacidad para desempeñar una función } \\
\text { productiva en diferentes contextos, conforme a } \\
\text { requerimientos de calidad del sector productivo. }\end{array}$ \\
\hline Leer & Planificar & $\begin{array}{l}\text { Cajero de local de comida rápida } \\
\text { Encargado de inventario } \\
\text { Escribir }\end{array}$ \\
$\begin{array}{l}\text { Trabajar en equipo } \\
\text { Expresión y comprensión oral }\end{array}$ & $\begin{array}{l}\text { Comunicación } \\
\text { Iniciativa y emprendimiento }\end{array}$ & Supervisor de operaciones logísticas \\
\hline
\end{tabular}

Fuente: Sence (2008).

una función laboral, según estándares definidos por el sector productivo. La Tabla 3 presenta el nivel de competencia de los 82 trabajadores evaluados en las empresas que participaron del estudio, independiente de la función que cumplen en cada una de ellas. Se puede observar en esta Tabla que las unidades de competencias laborales (UCL) transversal para todos los perfiles (Código SAO 001 o conductuales) tiene un promedio menor de cumplimiento $82,5 \%$ en comparación con el promedio de $96,2 \%$ de cumplimiento que tienen UCL Técnicas.

\section{Perfiles Ocupacionales Evaluados}

En la Tabla 4 se presenta el número total de perfiles evaluados en la muestra, distribución y recurrencia de esta, donde destaca que tanto las UCL conductuales como las UCL Técnicas bordean el

Tabla 3. Descripción de las unidades de competencias laborales (UCL) según empresas.

\begin{tabular}{lccccccc}
\hline \multicolumn{1}{c}{ Empresas } & & $\mathrm{n}$ & Media & Límite inferior & Límite superior & Mín. & Máx. \\
\hline \multirow{3}{*}{ Promedio UCL } & 1 & 37 & 78,259 & 77,177 & 79,341 & 63,4 & 81,9 \\
SAO 001 & 2 & 15 & 77,960 & 75,784 & 80,136 & 67,2 & 82,5 \\
& 3 & 20 & 78,825 & 78,391 & 79,259 & 76,0 & 80,0 \\
& 4 & 10 & 79,320 & 78,371 & 80,269 & 77,0 & 81,3 \\
& Total & 82 & 78,472 & & & 63,4 & 82,5 \\
\hline \multirow{2}{*}{ Promedio UCL } & 1 & 37 & 80,116 & 78,109 & 82,123 & 65,4 & 96,2 \\
Técnica & 2 & 15 & 79,693 & 77,231 & 82,156 & 75,9 & 93,8 \\
& 3 & 20 & 79,065 & 78,137 & 79,993 & 74,4 & 85,9 \\
& 4 & 10 & 79,100 & 77,998 & 80,202 & 75,8 & 80,7 \\
& Total & 82 & 79,659 & & & 65,4 & 96,2 \\
\hline
\end{tabular}

Tabla 4. Perfiles evaluados por empresa y su distribución.

\begin{tabular}{lcccccc}
\hline \multicolumn{1}{c}{ Perfil ocupacional } & \multicolumn{2}{c}{ Empresa } & Total & $\%$ \\
\cline { 2 - 5 } & 1 & 2 & 3 & 4 & & \\
\hline Embalador de fruta de exportación & 19 & 9 & 18 & 6 & 52 & 63,4 \\
Encargado cámara frigorífica & 1 & 0 & 0 & 0 & 1 & 1,2 \\
Encargado de bodega & 2 & 0 & 0 & 0 & 2 & 2,4 \\
Operador de armado y distribución de envases & 0 & 1 & 0 & 0 & 1 & 1,2 \\
Operador de ingreso y acondicionado de fruta & 2 & 0 & 0 & 0 & 2 & 2,4 \\
Operador grúa horquilla & 1 & 0 & 0 & 0 & 1 & 1,2 \\
Paletizador & 5 & 2 & 0 & 1 & 8 & 9,8 \\
Programador de línea de packing & 2 & 0 & 0 & 0 & 2 & 2,4 \\
Recepcionista & 2 & 0 & 0 & 0 & 2 & 2,4 \\
Supervisor de línea de proceso & 1 & 0 & 0 & 0 & 1 & 1,2 \\
Tarjador de packing & 2 & 3 & 2 & 3 & 10 & 12,2 \\
Total & 37 & 15 & 20 & 10 & 82 & 100 \\
\hline
\end{tabular}


$80 \%$ de cumplimiento, sin embargo, los promedios de estas últimas son superiores.

\section{Comparación Competencias Básicas, Conductuales y Funcionales}

$\mathrm{Al}$ interpretar ambas Unidades de competencias laborales en la Figura 1, se puede observar que tanto en la UCL Conductuales como en la UCL Técnicas existen muestras que escapan de los rangos o que se encuentran fuera de la media de las empresas, tanto a bajos como altos niveles de desempeño, es decir, algunos trabajadores se escapan del promedio grupal y tienen desempeños muy superiores, sin embargo, otros trabajadores presentan desempeños muy por debajo de la media grupal.

El número total de UCL evaluadas se presentan en la Figura 1, en la cual se observa que en la
Empresa 1 no hay diferencia significativa entre los niveles de competencias conductuales entre los candidatos de las distintas empresas, en la UCL conductuales (n.s. p >0,05 Tabla 5), salvo una leve tendencia a tener una mayor variabilidad de promedios de la media hacia el valor máximo, además, se pueden apreciar tres valores atípicos que en este caso recaen en los individuos $\mathrm{N}^{\circ} 16,28$ y 29 , los que poseen un valor muy bajo respecto de la media.

Tabla 5. Prueba homogeneidad de varianza en nivel de competencia de trabajadores en las cuatro empresas evaluadas.

\begin{tabular}{lcccc}
\hline & $\begin{array}{c}\text { Estadístico } \\
\text { de Levene }\end{array}$ & g11 & g12 & Sig. \\
\hline Promedio UCL SAO 001 & 2,904 & 3 & 78 & 0,04 \\
Promedio UCL Técnica & 3,233 & 3 & 78 & 0,027 \\
\hline
\end{tabular}

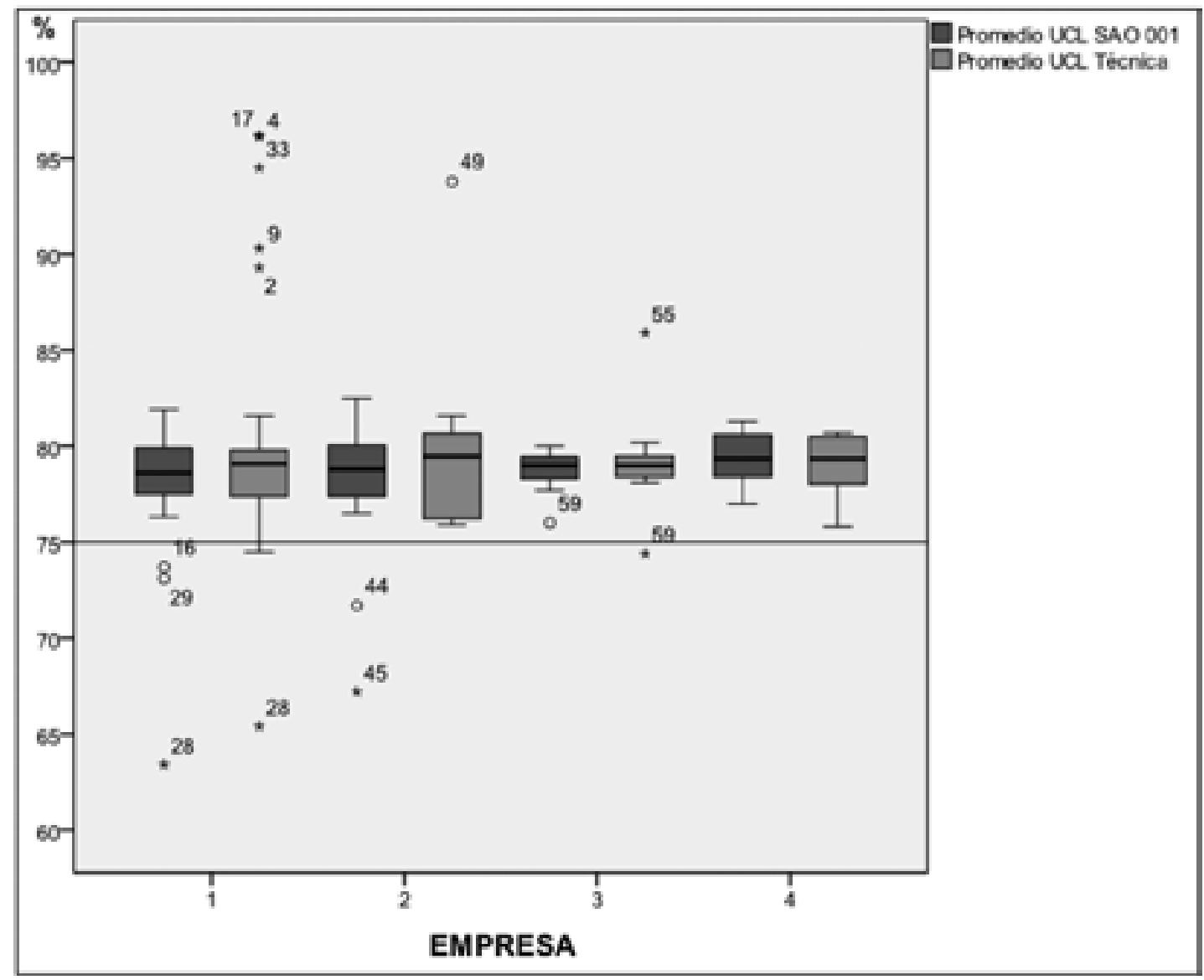

Figura 1. Nivel de competencia de empresas en UCL conductuales y técnicas. 
La Empresa 1 (E1), respecto de las UCL técnicas, muestra una tendencia a que los candidatos tienen un promedio mayor a las demás de la media hacia arriba, en cambio de la media hacia abajo hay una mayor variabilidad de promedios, encontrando muestras insuficientes según lo que exige la evaluación de competencia laboral. Se puede observar valores atípicos que sobrepasan los rangos máximos de las empresas como el individuo $\mathrm{N}^{\circ} 2,4,9,17$ y 33 , y el individuo $\mathrm{N}^{\circ} 28$, tiene un valor de la muestra insuficiente respecto de los demás de la empresa.

En relación con las UCL conductuales, la Empresa 2 (E2) muestra una mayor variabilidad de promedios con respecto a las demás, destacándose el promedio máximo de la muestra, también se puede observar dos muestras atípicas en los individuos $\mathrm{n}$ 44 y n 45 que tienen un promedio muy por debajo de la media de la empresa. Sin embargo, respecto de las UCL técnicas, la E2 tiene una mayor variabilidad de promedios, en general el $50 \%$ de las muestras tiene un valor por debajo del 79,6\%.

La Empresa 3 (E3) es la que tiene menos variabilidad en los valores de la muestra, observando muy poca distancia entre los valores mínimos y máximos, destacando solo un valor atípico que es el individuo n 59 tanto para UCL conductuales, como para las UCL técnicas, individuo que tiene un valor muy por debajo de la media en la empresa.

En la Empresa 4 se puede observar que no se presentan valores fuera de los rangos esperados tanto para UCL conductuales como para las UCL técnicas.

Cabe destacar que existen individuos que se caracterizan por poseer promedios bajos en las dos UCL evaluadas, como es el caso del individuo $\mathrm{N}^{\circ} 28$ de la E1, y el No 59 de la E3 que su promedio fue insuficiente tanto en la UCL conductual como la técnica, además se puede observar que hay trabajadores que se destacan notablemente en la UCL técnica, como los individuos $\mathrm{N}^{\circ} 2,4,9,17$ y 33 de la E1, el No 49 de la E2 y No 55 de la E3; en la E4 los datos no difieren estadísticamente entre sí y no hay muestras que escapen de los márgenes.

En la UCL SAO 001 o conductual, no se aprecia ningún individuo en las cuatro empresas con promedio superior que se escape de los rangos estadísticos.

No hay diferencias significativas entre los niveles de competencias de los candidatos de las distintas empresas, en las UCL técnicas evaluadas (n.s. p $>0,05$ Tabla 5)
El nivel de conocimientos generales y las capacidades intelectuales requeridos en la etapa posindustrial son superiores en la actualidad a aquellos exigidos por la utilización de las tecnologías más tradicionales. Niveles de escolaridad relativamente altos son capaces de asegurar que la fuerza de trabajo posea los rasgos personales, las habilidades intelectuales, comunicacionales y capacidad de responder a imprevistos que los nuevos procesos de producción requieren (Guernica Consultores S.A., 2009).

\section{Perfiles Ocupacionales Evaluados}

En esta investigación se tomaron en cuenta 52 de las 82 muestras que formaron parte del estudio, destacando el perfil de embalador de fruta de exportación, puesto que el resto pertenecen a otros perfiles ocupacionales y como se observa en el Tabla 4, este es el perfil que presenta una mayor recurrencia de la muestra entre las cuatro empresas.

\section{Perfil Ocupacional Embalador de Fruta de Exportación}

La formación basada en los perfiles de competencias constituye una señal de alto valor para el mercado del trabajo y las distintas instituciones vinculadas a este. Dentro de los usuarios de los estándares y perfiles están las instituciones de formación e instituciones de capacitación; organismos ya existentes que pueden usar estos para mejorar la calidad y pertinencia de sus servicios formativos (Fundación Chile, 2004).

En la Tabla 4 se destaca el perfil de embalador de fruta de exportación, que tiene una frecuencia de 52 veces en el proyecto, lo que equivale a un $63,4 \%$ del total de las muestras. Sin embargo, el análisis de varianza (Tabla 6) muestra que no hay diferencia significativa en los niveles de competencias entre los candidatos de las distintas empresas, tanto en la UCL SAO 001 como en la UCL Técnica evaluada.

En la Figura 2 se puede observar que las cuatro empresas son homogéneas en nivel de competencias conductuales de sus trabajadores, para este perfil, siendo la E2 la que presenta mayor variabilidad en promedios de las muestras. Existen dos datos que se escapan de los márgenes, siendo uno de ellos, el individuo $\mathrm{N}^{\circ} 11$ de la E1 y su nivel de competencia es muy bajo respecto de la media, además se encuentra bajo el promedio que se exige 
Tabla 6. Prueba homogeneidad de varianza en el nivel de competencia en perfil embalador de fruta de exportación en las 4 empresas evaluadas.

\begin{tabular}{lcccc}
\hline & Estadístico de Levene & gl1 & g12 & Sig. \\
\hline Promedio UCL SAO 001 & 2,128 & 3 & 48 & 0,109 \\
Promedio UCL Técnica & 1,866 & 3 & 48 & 0,148 \\
\hline
\end{tabular}

(75\%), por lo que es denominado "candidato aún no competente" en su labor. El candidato $\mathrm{N}^{\mathrm{o}} 36$, a pesar que se observa una marcada diferencia con respecto a su nivel y al resto de las muestras de la empresa, está por sobre el $75 \%$ exigido para ser un candidato "competente" en su labor.

En la Figura 2 se puede observar, respecto de las UCL técnicas evaluadas, una mayor homogeneidad en los promedios entre las empresas que participaron del proyecto; se puede destacar candidatos que sobresalen en su labor con promedios muy superiores a la media de las empresas, como los individuos $\mathrm{N}^{\circ} 12 \mathrm{yN}^{\circ} 4$ de la E1, el individuo $\mathrm{N}^{\circ} 25$ de la E2 y el $\mathrm{N}^{\mathrm{o}} 32$ de la E3. También se observa que hay candidatos "Aún no competentes" en la E1, que están por debajo del $75 \%$ exigido, y el candidato $\mathrm{N}^{\circ} 36$ de la E3 que aparte de tener un promedio inferior a este porcentaje, muestra una notable diferencia con el resto de las muestras de la misma empresa.

Según Ministerio del Trabajo y Previsión social (2008), toda persona podría solicitar la certificación de sus competencias laborales según el sistema que establece la ley, y sin que ésta constituya obligación o requisito para desempeñar una determinada actividad. La certificación será otorgada mediante entidades acreditadas a través

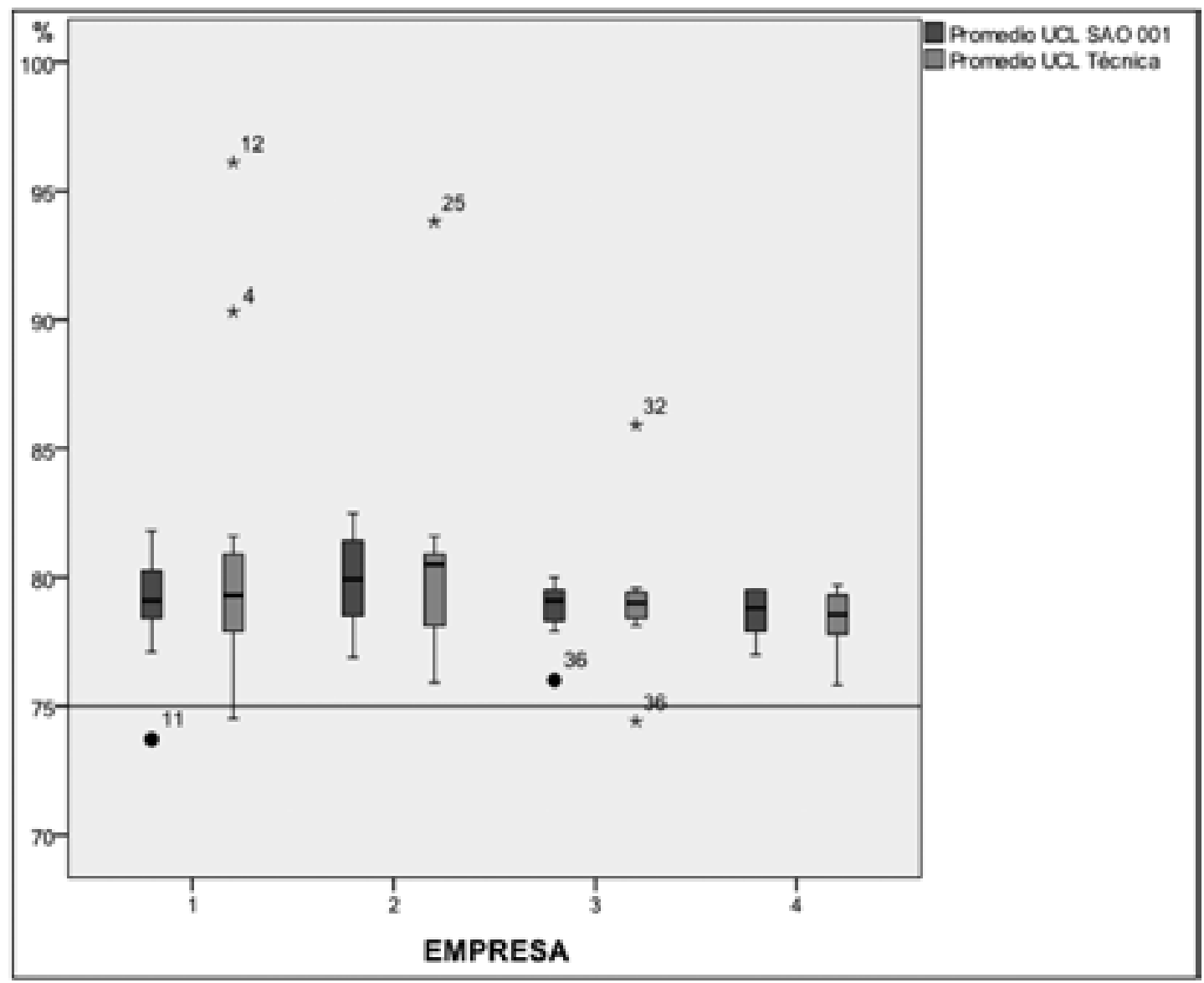

Figura 2. Nivel de competencia perfil embalador de fruta de exportación UCL conductuales y técnica. 
de un marco metodológico común aceptado por los distintos sectores productivos.

\section{Conclusiones}

A partir del trabajo realizado se puede concluir lo siguiente:

No existen diferencias significativas entre los niveles de competencia de trabajadores de las empresas agrícolas participantes del proyecto, independiente de la función que cumplen dentro de ellas, así como tampoco entre las competencias de los trabajadores que ejecutan un mismo perfil ocupacional en empresas diferentes y que han sido partícipe del programa de evaluación de competencias laborales temporada 2010-2011, en la VI Región del Libertador Bernardo O'Higgins.

En el análisis de la UCL conductual SAO 001 se pudo observar que el $93,3 \%$ de los trabajadores muestran un nivel de competencia mayor al $75 \%$ que exige el programa de certificación de competencias laborales.

En el análisis de las UCL técnicas se pudo observar que el $95,1 \%$ de los trabajadores muestran un nivel de competencia mayor al $75 \%$ que exige el programa de certificación de competencias laborales.
Por lo tanto, un $4,9 \%$ no reúne las competencias necesarias para ser considerado un candidato competente en su labor

Los resultados muestran que existen trabajadores en las cuatro empresas que destacan claramente en sus competencias técnicas, pero que solo cumplen con las mínimas exigencias que se le piden en la UCL.

Los resultados muestran que un $92,6 \%$ de los candidatos son considerados competentes dentro del programa de certificación de competencias laborales. El 7,4\% muestran brechas en el aspecto de higiene y seguridad.

El perfil de embalador de fruta de exportación representa un $63,4 \%$ del total de la muestra, lo que destaca la relevancia de este perfil en las empresas del sector.

En el perfil de embalador de fruta de exportación, un $98 \%$ de las muestras está por sobre el $75 \%$ exigido por el programa de certificación de competencias laborales, respecto de la UCL transversal. El 2\% restante constituye candidatos aún no competentes en dicha labor.

El 96,1\% del perfil de embalador de fruta de exportación está por sobre el $75 \%$ que exige el programa de certificación de competencias laborales, respecto de la UCL técnica.

\section{Literatura Citada}

Arguelles, A. (compilador).

1999. Hacia una educación permanente en Chile. Santiago. Chile.76 p.

De Ferranti D.; Perry G.; Gill I.; Maloney W.; Guasch J.; Sánchez-Páramo C.; Schady N.

2002. Cerrando la brecha en educación y tecnología. Bogotá, Colombia. 216 p.

Etiennette, I.

2002. Hacia una educación permanente en Chile. Santiago, Chile. 76 p.

Fundación Chile.

2004. Competencias laborales para Chile 1999-2004. Capital humano: productividad y desarrollo de las personas. Santiago. Chile. 146 p.

Fundación Chile.

2007. Competencias laborales: Industria. Láctea. Santiago. Chile. 92 p.
Fundación Chile.

2010. Manual competencias laborales. Santiago. Chile. 50 p

Fundación para el Desarrollo Frutícola (FDF).

2010. Ponderación instrumentos evaluación según perfil ocupacional. Santiago, Chile. 2 p.

Ministerio del Trabajo y Previsión Social.

2008. Ley-20267. Sistema nacional de certificación de competencias laborales. Santiago, Chile.17 p.

Servicio Nacional de Capacitación y Empleo (SENCE).

2008. Modelo de competencias laborales y el sistema nacional de certificación de competencias laborales. Santiago, Chile. $24 \mathrm{p}$.

Guernica Consultores S.A.

2009. Contratación de servicios destinados a evaluar impacto de la línea de certificación de competencias laborales programa Chile Califica. Santiago, Chile. 208 p. 
